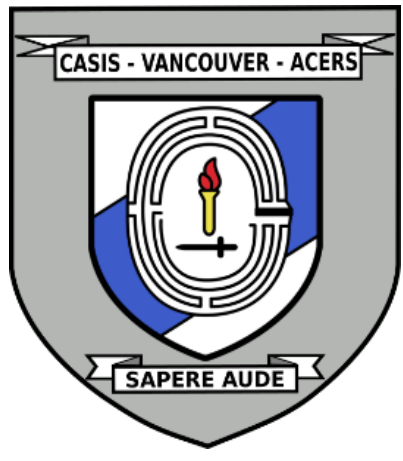

\title{
GLOBAL INFORMATION AND DIGITALIZED INFLUENCE IN A DATA- DRIVEN WORLD
}

Date: November 24, 2021

Disclaimer: This briefing note contains the encapsulation of views presented by the speaker and does not exclusively represent the views of the Canadian Association for Security and Intelligence Studies.

\section{KEY EVENTS}

On November 24, 2021, Dr. Emma Briant presented Global Information and Digitalized Influence in a Data-driven World at the 2021 CASIS West Coast Security Conference. The primary concepts of Dr. Briant's presentation centered on the types of tactics and methodologies used by Cambridge Analytica during political campaigns and how these methodologies have been used to drive extremism. Dr. Briant's presentation was followed by a question and answer period directed at a group of panelists allowing the audience and CASIS Vancouver executives to directly engage with the content of each speaker's presentation.

\section{NATURE OF DISCUSSION}

\section{Presentation}

Dr. Briant's presentation began with a brief history of the Cambridge Analytica scandal and detailed how their data analytics relied heavily on the personality trait, neuroticism, to target individuals with fear-driven messaging. Dr. Briant presented several examples of how this approach has affected society, including the techniques used behind political campaigns and strategies used by anti-vax groups to perpetuate disinformation. Dr. Briant suggested the government may not have the best approach to counter these fear-driven disinformation campaigns and provided a potential solution on how to better address the situation. 


\section{Question Period}

The question period highlighted the difficulty of addressing echo-chambers in the online environment and how social media tools and algorithms are used in the creation and amplification of echo-chambers.

\section{BACKGROUND}

\section{Presentation}

Dr. Briant's presentation began with a brief history of the Cambridge Analytica scandal in which they harvested 18 million Facebook profiles that were obtained non-consensually. They used the OCEAN personality test and modeled it onto these Facebook profiles to evaluate users' Openness, Conscientiousness, Extraversion, Agreeableness, and Neuroticism. Through their analytics, Cambridge Analytica identified Neuroticism as an indicator of anxiety and tendency to worry and fear, so they began focusing their attention on how to activate people using such triggers. They discovered that they were getting substantially more engagement from those who scored higher in neuroticism when pushing fear-driven or extremist messaging.

Dr. Briant then turned her focus on a project she had worked on previously, regarding what was being done with church data behind US political campaigns. In conjunction with a religious app company, Cambridge Analytica was retained by the Philanthropy Roundtable organization to produce a church survey and Facebook micro-targeting campaign so they could reach their congregations. It was discovered that sensitive data obtained from this religious app was being marketed for political purposes. More specifically, they were looking at how to move certain groups of people towards voting Republican.

The religious modeling behind these political campaigns served to target those high in neuroticism and set them aside specifically for fear-driven campaigns. The Crooked Hillary Campaign arose as a means to suppress voters by spreading conspiracy theories and inciting fear in those who were higher in neuroticism. More recently, paranoia-based operations, such as the Stop the Steal Campaign, seem to be targeting those with mental health challenges, many of whom were activated in a very aggressive way at the Capitol Hill riot.

Dr. Briant emphasized the importance of identifying how anxiety is used in these types of campaigns. There has been a worrying number of veterans and military personnel being targeted by these campaigns, which can become a significant 
security concern. In some countries, individuals are being encouraged to selfdeclare their mental health challenges in an effort to be supportive. However, Dr. Briant stressed that one must be extremely cautious when it comes to individuals who have important positions or those with particular skill sets that could be targeted by foreign entities and domestic extremists.

At this point in the presentation, Dr. Briant highlighted a clear parallel between the tactics being used by Cambridge Analytica behind political campaigns and the tactics used by anti-vaxxers to drive disinformation. In her research, Dr. Briant discovered that the lead psychologist behind Cambridge Analytica, Patrick Fagan, had also been working with an anti-vaxxer group, HART, on their data operations and messaging strategies. In leaked chats, Mr. Fagan justified using similar behavioural techniques to target people they want to pull over to the antivax side by provoking fear among neurotics. In his leaked chats, he discussed how those who advocate for mandatory face masks are neurotic and, therefore, messaging should highlight that wearing masks is harmful and unfair.

To conclude, Dr. Briant expressed her concern about these paranoia operations that attempt to isolate the people that are already prone to have anxiety about particular issues. The way our governments are responding to these issues may not be the most effective approach. Rather than focusing on correcting the falsehoods being perpetuated by anti-vax groups, the government should focus on privacy legislation and preventing companies like Cambridge Analytica from gaining access to this type of data. We need to tackle how these companies are reaching people and change the nature of the debate. Dr. Briant argued that we must discuss the facts in a reasonable, educated environment and ensure the facts are not morphed into something that is designed to spread fear, suppress votes, or make us abandon reliable media sources.

\section{Question Period}

During the question and answer period, the audience asked how we can tackle the challenge of echo-chambers that polarize members of society, especially given the increased internet usage brought on by the pandemic. Dr. Briant clarified that it is not so much the echo-chambers themselves, but about how these echo-chambers get created. The way individuals get pulled into these rabbit holes and echo-chambers is largely the result of social media companies' algorithms.

Dr. Briant deconstructed the issue into two layers. On one side, there are the organizations or actors creating the content to target various groups of people and 
spread their message using social media. As with her Cambridge Analytica study, they had created a list of neurotic individuals they wanted to target by using Facebook's 'custom audience' feature. This enabled them to only target specific types of people without being flagged by Facebook. On the other side of the issue, there are social media algorithms designed to identify 'look-alike' audiences, amplifying similar content to people who continue to actively engage with the messaging.

Dr. Briant noted that currently, there is a large focus on the social media companies and their algorithms; however, there is little mention about these types of 'audience' tools that enable organizations or actors to target specific individuals with their content. Having these tools available in the first place, is reinforcing these rabbit holes and online radicalization. In order to effectively tackle this issue, Dr. Briant argued that the use of these types of tools needs to be disabled in addition to addressing social media companies' algorithms.

\section{KEY POINTS OF DISCUSSION}

\section{Presentation}

- Companies such as Cambridge Analytica have been employing questionable behavioural approaches to 'activate' people based on fear.

- Through their research, Cambridge Analytica identified neuroticism as an indicator of anxiety and tendency to worry, so they began pushing fear-driven or extremist messaging to gain higher engagement.

- Fear-driven political campaigns such as the Crooked Hillary Campaign and the Stop the Steal Campaign were designed to instill fear and suppress votes and incite people into violent action, as could be seen in the Capitol Hill riot.

- Similar tactics have also been used by anti-vax groups, who have been targeting neurotics with fear-driven messaging to persuade them to join their cause.

- Governments should tackle this issue by focusing on privacy legislation and preventing companies like Cambridge Analytica from gaining access to this type of data in the first place rather than trying to correct falsehoods.

\section{Question Period}

- In order to tackle the polarizing nature of echo-chambers, more focus needs to be placed on social media algorithms and the tools that enable selective targeting of individuals. 
- Cambridge Analytica had been using Facebook's 'custom audience' feature to selectively target neurotics with fear-driven messaging, without being flagged by Facebook.

- Once content is shared, Facebook's algorithm then amplifies similar content to people who continue to actively engage with the messaging, thus facilitating echo-chambers.

- By disabling access to features such as Facebook's 'custom audience,' we can curb the escalation of echo-chambers more effectively.

\section{@} Commercial-NoDerivatives 4.0 International License.

(C) (EMMA BRIANT, 2022)

Published by the Journal of Intelligence, Conflict, and Warfare and Simon Fraser University

Available from: https://jicw.org/ 